\title{
DNA methyltransferase 1 functions through C/ebpa to maintain hematopoietic stem and progenitor cells in zebrafish
}

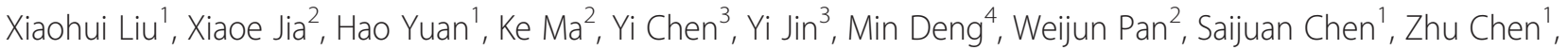
Hugues de The ${ }^{1,5}$, Leonard I Zon', Yi Zhou ${ }^{6 *}$, Jun Zhou ${ }^{1 *}$ and Jun Zhu ${ }^{1,5^{*}}$

\begin{abstract}
Background: DNA methyltransferase 1 (Dnmt1) regulates expression of many critical genes through maintaining parental DNA methylation patterns on daughter DNA strands during mitosis. It is essential for embryonic development and diverse biological processes, including maintenance of hematopoietic stem and progenitor cells (HSPCs). However, the precise molecular mechanism of how Dnmt1 is involved in HSPC maintenance remains unexplored.
\end{abstract}

Methods: An N-ethyl-N-nitrosourea (ENU)-based genetic screening was performed to identify putative mutants with defects in definitive HSPCs during hematopoiesis in zebrafish. The expression of hematopoietic markers was analyzed via whole mount in situ hybridization assay (WISH). Positional cloning approach was carried out to identify the gene responsible for the defective definitive hematopoiesis in the mutants. Analyses of the mechanism were conducted by morpholino-mediated gene knockdown, mRNA injection rescue assays, antiphosphorylated histone $\mathrm{H} 3(\mathrm{pH} 3)$ immunostaining and TUNEL assay, quantitative real-time PCR, and bisulfite sequencing analysis.

Results: A heritable mutant line with impaired HSPCs of definitive hematopoiesis was identified. Positional cloning demonstrated that a stop codon mutation was introduced in dnmt1 which resulted in a predicted truncated Dnmt1 lacking the DNA methylation catalytic domain. Molecular analysis revealed that expression of CCAAT/enhancer-binding protein alpha (C/ebpa) was upregulated, which correlated with hypomethylation of CpG islands in the regulation regions of cebpa gene in Dnmt1 deficient HSPCs. Overexpression of a transcriptional repressive SUMO-C/ebpa fusion protein could rescue hematological defects in the dnmt1 mutants. Finally, dnmt1 and cebpa double null embryos exhibited no obvious abnormal hematopoiesis indicated that the HSPC defects triggered by dnmt1 mutation were C/ebpa dependent.

Conclusions: Dnmt1 is required for HSPC maintenance via cebpa regulation during definitive hematopoiesis in zebrafish.

Keywords: Dnmt1, C/ebpa, HSPCs, Zebrafish

\footnotetext{
*Correspondence: yzhou@enders.tch.harvard.edu; junjun_j@yahoo.com; zhuj1966@yahoo.com

${ }^{6}$ Stem Cell Program, Hematology/Oncology Program at Children's Hospital Boston, Harvard Medical School, Boston, MA 02114, USA

${ }^{1}$ CNRS-LIA124, Sino-French Research Center for Life Sciences and Genomics, State Key Laboratory of Medical Genomics, Rui Jin Hospital, Shanghai Jiao

Tong University School of Medicine, Shanghai 200025, China

${ }^{5}$ Equipe Labellisée No. 11 Lique Nationale Contre le Cancer, Hôpital St. Louis, Université de Paris 7/INSERM/CNRS UMR 944/7212, 75475 Paris, France

Full list of author information is available at the end of the article
} 


\section{Background}

In vertebrates, hematopoiesis takes place in two consecutive waves, primitive and definitive ones [1,2]. Primitive hematopoiesis, also known as embryonic hematopoiesis, predominantly produces erythroid and myeloid cells [3,4]; while definitive hematopoiesis, also called adult hematopoietic wave, generates hematopoietic stem cells (HSCs) that are capable of producing all lineages of blood [5]. The zebrafish (Daniorerio) is an excellent genetic system for the study of hematopoietic development [6,7], especially by characterization of thousands of mutants isolated from large-scale forward genetic screens [8,9]. In zebrafish, definitive HSCs arise from the ventral wall of the dorsal aorta (VDA), the zebrafish equivalent of the aorta/gonad/mesonephros (AGM) of mammals [10,11], then HSCs migrate through the caudal hematopoietic tissue (CHT) to the thymus and kidney marrow [12], where adult hematopoiesis occurs, similar to HSC migration through fetal liver and home to bone marrow in mammals.

The molecular regulation of hematopoiesis includes interactions of lineage-specific transcription factors and a series of epigenetic modifications, such as DNA methylation and covalent histone tail modifications [13]. DNA methylation is an important epigenetic regulation mechanism that regulates normal development through influencing gene transcription, genomic imprinting, and genome stability in mammal cells [14-16]. In hematologic malignancies, dysregulation of DNA methylation may result in global shifts in gene expression, which frequently leads to increased self-renewal in malignant blood cells at the expense of normal differentiation [17].

Three active DNMTs, namely DNMT1 [18], DNMT3A, and DNMT3B $[19,20]$, have been identified in mammals. DNMTs are highly evolutionarily conserved with a regulatory region attached to a catalytic domain [21]. DNMT1 is the most abundant DNA methyltransferase in mammalian cells and considered to be the key maintenance methyltransferase [22]. In mammals, DNMT1 null mutant embryonic stem cells are viable and contain a small percentage of methylated DNA and methyltransferase activity [23]. Mouse embryos homozygous for a deletion of Dnmt1 die at 10 to 11 days gestation due to development defects [24]. Reduced Dnmt1 activity in xenopus [25] and zebrafish [26,27] has similar consequences. Dnmt1 also plays important roles in HSPCs. The deletion of Dnmt1 has no influence on the mature cells in the hematopoietic system but causes decreased niche retention and self-renewal and differentiation defects of HSPCs [28]. In acute myeloid leukemia (AML), the expression of DNMTs is upregulated [29]. Conditional knockout of Dnmt1 blocks development of leukemia, and haploinsufficiency of Dnmt1 is sufficient to delay progression of leukemogenesis and impair leukemia stem cell (LSC) self-renewal without altering normal hematopoiesis [30]. The precise mechanism of the
Dnmt1 regulation of HSC function requires further investigation.

In this study, a heritable zebrafish mutant line with hematopoietic defects identified through ENU-based forward genetic screening was found defective in dnmt1 gene. Phenotype characterization of dnmt1 mutant has uncovered severely impaired definitive hematopoiesis. Further molecular mechanistic studies revealed that cebpa was a Dnmt1 downstream target gene and activated as a result from hypomethylation of its regulation regions in dnmt1 mutants, which suggested cebpa was a key downstream target of dnmt1 gene in HSPCs. We further demonstrated that the elevated C/ebpa activity was required and accounted for, at least in part, the defective definitive hematopoiesis.

\section{Results}

Zebrafish mutant line Idd794 displays impaired definitive hematopoiesis

To search for novel genes involved in regulating definitive hematopoiesis, we established an ENU-based genetic screening strategy to identify putative mutants with defects in definitive HSPCs in zebrafish. Whole-mount mRNA in situ hybridization (WISH) analysis of cmyb [31], a marker of HSPCs, was used to screen for mutants. In the mutant line ldd794, cmyb expression in homozygous embryos was reduced from 36 hours post-fertilization (hpf) in the AGM (Figure 1A, B) and was almost absent at 5 days post-fertilization (dpf) in the CHT (Figure 1I, J). Similarly, the expression of two other HSPC markers runx1 as well as $s c l$ were also decreased (Figure $1 \mathrm{~K}-\mathrm{N}$ ), suggesting the definitive HSPCs were impaired. ldd794 heterozygous fish was crossed with a Tg (cmyb:EGFP) homozygous individual (a stable zebrafish transgenic line expressing EGFP under the control of the cmyb promoter) [32]. The adult fishes carrying both dnmt1 mutant allele and EGFP transgene were incrossed. As expected, the number of EGFP-positive cells was significantly decreased in the CHT of approximate $25 \%$ EGFP positive offspring at $4 \mathrm{dpf}$ (Additional file 1: Figure S1A). This result further confirmed the HSPCs were specifically affected in ldd794 mutants.

The temporal and spatial expression patterns of a set of hematopoietic transcription factors and key genes involved in either lineage determination or differentiation were examined in ldd794 mutants. The expression of erythrocyte progenitor marker gata1 [33], mature erythrocyte marker hbae1 [34], myeloid-specific marker mpx [35], l-plastin [36] and lysozyme C [37], and lymphoidspecific marker rag1 [38] were all diminished in ldd794 homozygous mutants (Figure 1O-Z). The decrease of multiple hematopoietic lineages suggested that the deficiency of hematopoietic precursors and/or progenitors occurred in the dnmt1 mutants. 

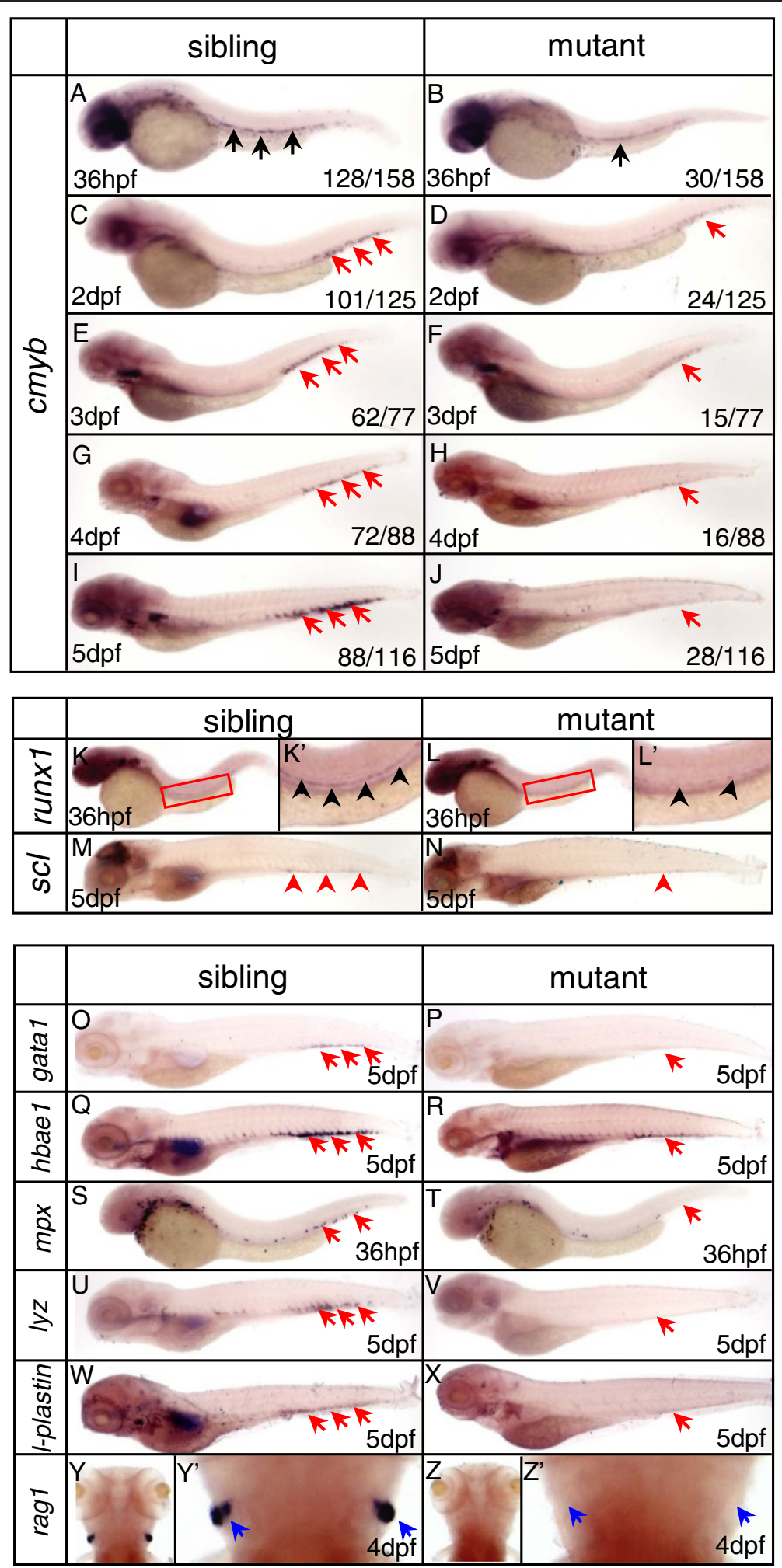

Figure 1 (See legend on next page.) 
(See figure on previous page.)

Figure 1 Impairment of definitive hematopoiesis in dnmt1 mutant zebrafish. (A-J) WISH analysis of cmyb expression from $36 \mathrm{hpf}$ to 5 dpf. In Idd794 mutant, cmyb expression was decreased from 36 hpf (A-B) and absent at 5 dpf (I-J). (K, K', L, L') Expression of hematopoietic progenitor marker runx 1 was decreased at 36 hpf. (K', L') Magnified images of the boxed regions in $K$ and $L$, respectively. (M-N) Expression of hematopoietic progenitor marker scl was decreased at $5 \mathrm{dpf}$. (O-Z) WISH analysis of key hematopoietic markers in dnmt1 mutant embryos and wild-type siblings. Expression of erythrocyte progenitor marker gata $\mathbf{( O} \mathbf{O}-\mathbf{P})$, mature erythrocyte marker hbael $(\mathbf{Q}, \mathbf{R})$, myeloid-specific marker mpx (S, T), macrophage marker lyz (U, $\mathbf{V})$, I-plastin (W, X) were decreased. Expression of lymphocyte maker rag $1\left(\mathbf{Y}, \mathbf{Y}^{\prime}, \mathbf{Z}, \mathbf{Z}^{\prime}\right)$ was completely absent at $4 \mathrm{dpf}$. Blue arrows indicate the position of the thymus; red arrows indicate the CHT; black arrows indicate the AGM.

To exclude the possibility that the defective definitive hematopoiesis was due to the preexisting primitive hematopoietic defects in ldd794 mutants, a series of markers involved in primitive hematopoiesis, such as scl, hbae1, pu.1, mpx, and lysozyme $C$ were also examined at 22 hpf. No overt changes have been detected (Additional file 1: Figure S1B), suggesting that primitive hematopoiesis was not affected in the dnmt1 mutants.

Since normal vasculogenesis is required for the birth of HSCs from the ventral wall of the dorsal aorta in the zebrafish embryo $[10,11,39,40]$, we evaluated early vascular development by expression of vascular markers. No obvious differences in $f l k 1$ and ephrinB2 expressions were observed between ldd794 siblings and mutants (Additional file 1: Figure S1C), suggesting that the vascular system and artery-vein differentiation remained intact and the hematopoiesis defects were not due to impaired vasculature.

\section{Zebrafish Idd794 mutant encodes a truncated Dnmt1 lacking the enzymatic catalytic domain}

Positional cloning approach was applied to identify the gene responsible for the defective definitive hematopoiesis in ldd794 mutants. Bulk segregation analysis indicated that the potential mutation site was located on chromosome 3 . The 528 putative mutant embryos (1,056 meioses) were further examined. Seven homologous recombinations of sslp1 (zC250L3) and eight homologous recombinations of sslp2 (zC74M13) from different embryos were identified, which enabled us to localize the mutation to a $0.45-\mathrm{MB}$ region containing eight genes (Figure 2A). Complementary DNA (cDNA) sequencing of all eight candidate genes revealed that in dnmt1, there was a $\mathrm{T}$ to $\mathrm{A}$ transversion, which introduced a stop codon at 743th amino acid, resulting in a predicted truncated Dnmt1 lacking the DNA methylation catalytic domain (Figure 2B-D). The gene synteny analysis revealed that Dnmt1 genomic context was highly conserved from zebrafish to human (Figure 2E).

\section{Loss of Dnmt1 catalytic activity leads to specific HSPC defects}

WISH analysis revealed that zebrafish dnmt1 was expressed ubiquitously, including the hematopoietic regions (Additional file 1: Figure S2). The dnmt1 specific antisense ATG-morpholino (MO) was injected into wild- type embryos at one-cell stage to reproduce the phenotypes observed in ldd794 mutant embryos. As expected, the expression of $c m y b$ diminished from $36 \mathrm{hpf}$ (Figure 3B, $\left.B^{\prime}\right)$ in the AGM and significantly reduced in the CHT at 5 dpf (Figure 3F, F'), which was exactly a phenocopy of ldd794 mutants. Unsurprisingly, erythrocytes, myeloid cells, and lymphocytes were decreased in dnmt1 ATG morphants (Additional file 1: Figure S3). Furthermore, a dnmt1 splicing $\mathrm{MO}$, specifically affecting splicing of precursor dnmt1 RNA transcripts (Additional file 1: Figure S4), was also able to mimic the Dnmt1 protein truncation identified in ldd794 mutants. A similar effect to that of ATG MO was observed (Figure 3C, C', G, G'), further confirming that the loss of Dnmt1 catalytic activity indeed specifically led to observed HSPC defects in the dnmt1 mutants.

Finally, specific in vivo rescue experiments were carried out. Full-length zebrafish dnmt1 mRNA was coinjected with dnmt1 ATG MO into wild-type embryos. The results showed that dnmt1 mRNA could efficiently rescue the hematopoietic defects in dnmt1 morphants (Figure 3D, D', H, H'). In contrast, co-injection of the truncated dnmt1 mutant mRNA with the dnmt1 ATG MO was ineffective (data not shown). Consistently, dnmt1 mRNA could also restore the defects of HSPCs in ldd794 mutant embryos (Figure 3I-N). These data demonstrated that the phenotype observed in dnmt1 mutants was indeed Dnmt1 dependent.

To investigate whether the observed deficient Dnmt1mediated phenotypes were due to abnormal cell proliferation or apoptosis of definitive HSPCs, the antiphosphorylated histone $\mathrm{H} 3(\mathrm{pH} 3)$ immunostaining and TUNEL assay were performed, respectively. A decrease of pH3 and cmyb-EGFP double positive cells was detected while no obvious change of TUNEL and cmyb-EGFP double positive cells was found in dnmt1 mutants (Figure 3O-U, Additional file 1: Figure S5). Similar results were observed in dnmt1 morphants (data not shown), suggesting that the defects of HSPCs in dnmt1-deficient embryos were caused by decreased proliferation.

\section{Increased cebpa expression was correlated with hypomethylation of $\mathrm{CpG}$ islands}

Given the fact that Dnmt1 predominantly methylates hemimethylated $\mathrm{CpG}$ dinucleotides in the mammalian 


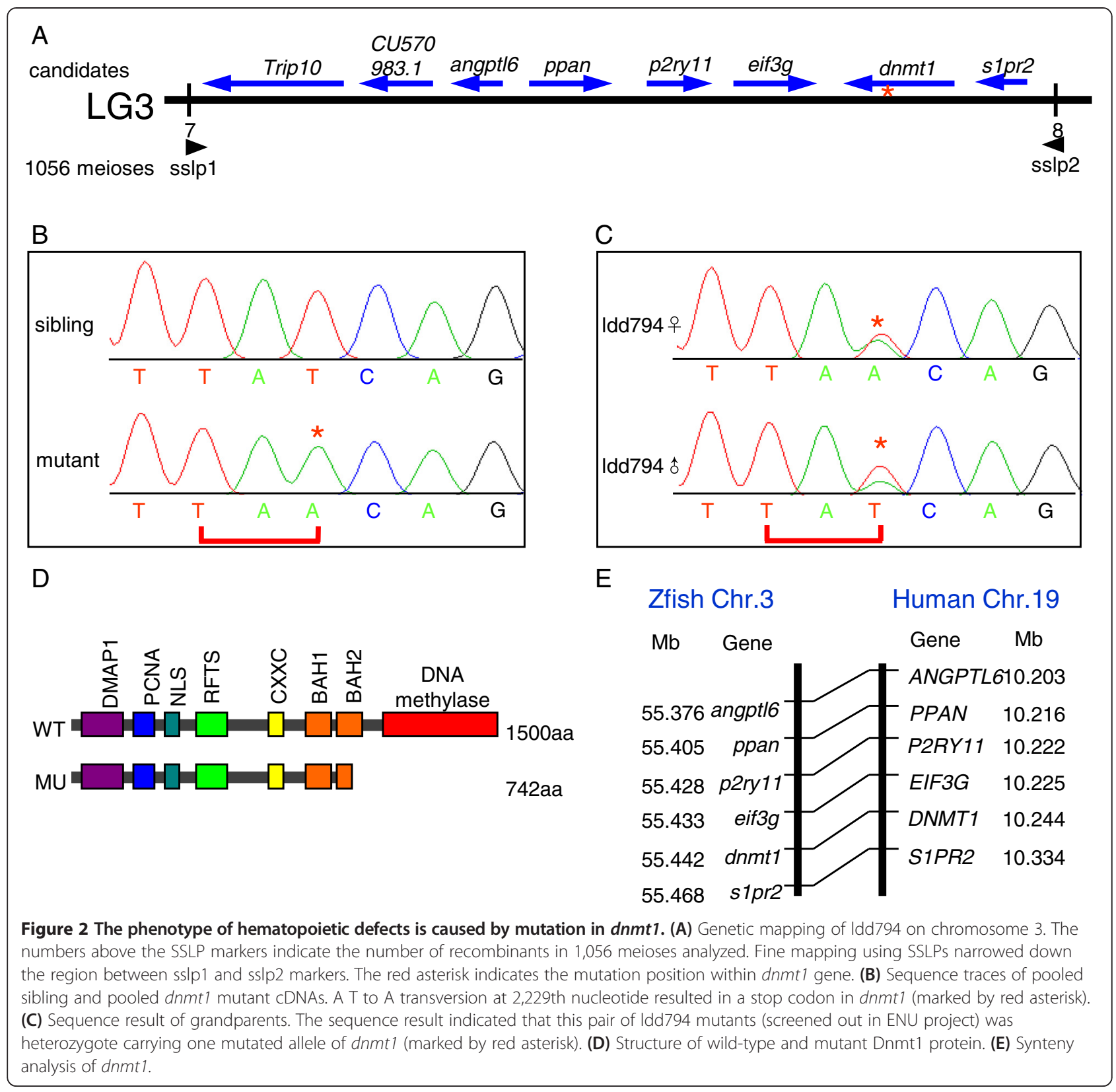

genome and facilitates repression in promoter regions, we speculate that the loss of Dnmt1 might activate some key negative regulators of definitive hematopoiesis.

C/ebpa, a member of the basic leucine zipper protein family of transcription factors $[41,42]$, not only plays a pivotal role in granulopoiesis [43] but also regulates the selfrenewal and proliferation of HSPCs at a much earlier stage during mouse hematopoiesis [44-47]. C/ebpa deficiency leads to hyperproliferation and increased selfrenewal capacity in both fetal and adult HSCs $[44,45,47]$, while activation of C/ebpa is sufficient to repress stem cell capacities and proliferation of HSCs [46]. More direct evidence is that $\mathrm{C} / \mathrm{EBPa}$ has previously been reported as a cell-cycle inhibitor [48]. These evidences prompted us to test whether C/ebpa function was important for mediating the observed HSPC proliferation phenotypes in the dnmt1 mutants.

Firstly, to test whether cebpa was upregulated in dnmt1-deficient HSPCs, dnmt1 MO was injected into Tg ( $c m y b$ :EGFP) embryos at one-cell stage, then $c m y b$ EGFP positive cells were sorted and collected at $3 \mathrm{dpf}$ (Figure 4A). RT-PCR results showed that the expression of cebpa indeed increased in dnmt1-deficient $c m y b$-EGFP positive cells (Figure 4B).

Secondly, we checked the DNA methylation status of the CpG islands located near the zebrafish cebpa gene. 

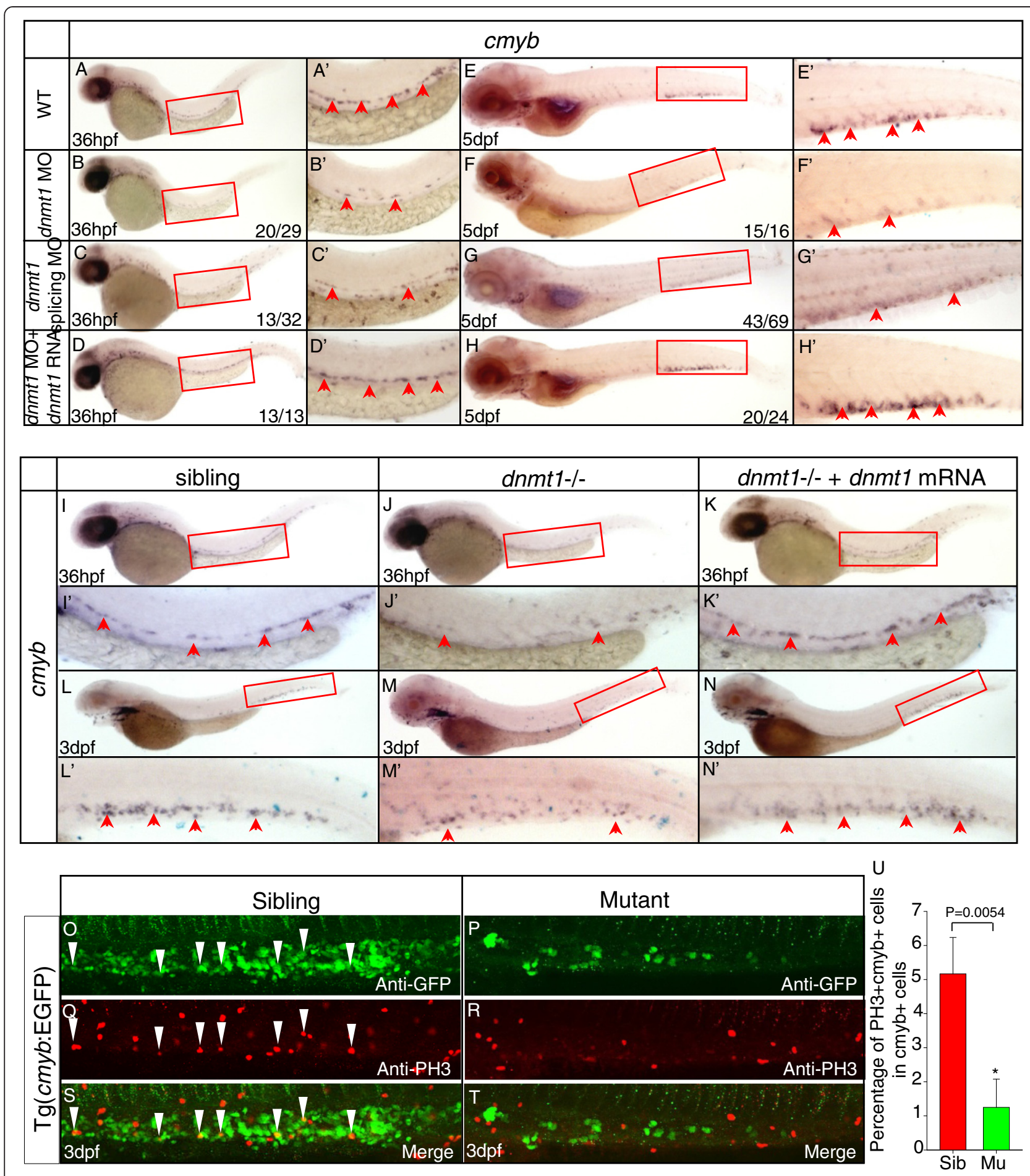

Figure 3 Dnmt1 mRNA rescue and anti-phosphorylated histone $\mathrm{H3}(\mathrm{pH} 3)$ immunostaining assays in dnmt1-deficient embryos.

(A-H) Dnmt1 mRNA rescue assay at $36 \mathrm{hpf}$ (A-D) and $5 \mathrm{dpf}$ (E-H). Dnmt1 ATG morpholino (4 ng) and splicing-specific morpholino (8 ng) effectively reproduced decreased cmyb expression phenotype in wild-type embryos (B, C, F, G). Dnmt1 mRNA rescued the defects of dnmt1 morphants. (I-N) Rescue assay of wild-type dnmt1 mRNA in Idd794 dnmt1 mutants at 36 hpf (I-K) and 3 dpf (L-N). Wild-type dnmt1 mRNA effectively restored cmyb expression in Idd794 dnmt1 mutants at $36 \mathrm{hpf}(\mathrm{K})$ and $3 \mathrm{dpf}(\mathrm{N})$. ( $\left.\mathbf{A}^{\prime}-\mathbf{N}^{\prime}\right)$ Magnified images of the boxed regions in $\mathbf{A}$ to $\mathbf{N}$, respectively. Red arrows indicate cmyb-positive HSPCs in the AGM or CHT. (O-T) The proliferation of HSPCs was reduced in dnmt1-/mutants. Double immunostaining of cmyb-EGFP (O-P) and anti-PH3 (Q-R) in the CHT of Tg(cmyb-EGFP) line at 72 hpf. The bottom panel shows merged images (S, T). (U) Statistics of PH3 and cmyb-EGFP double positive cells. 


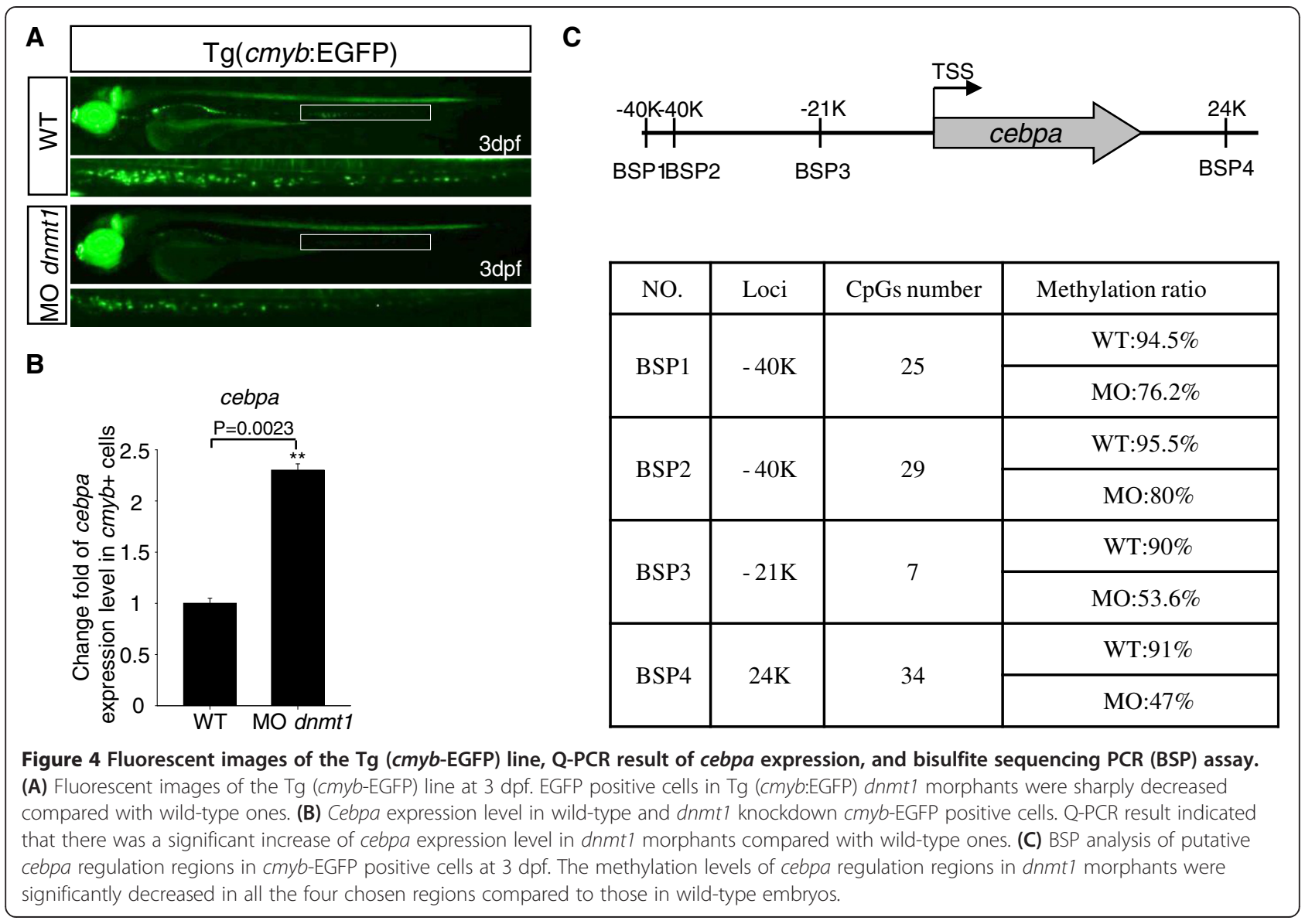

Up to $40 \mathrm{~kb}$ long of upstream, the coding region, and the downstream genomic sequence of the zebrafish cebpa gene were searched for potential CpG islands. A total of 14 candidate $\mathrm{CpG}$ islands were found, and their DNA methylation statuses were evaluated by bisulfite sequencing analysis. The hypermethylation was found in four CpG island regions (BSP1 to BSP4) near the cebpa gene in wild-type $c m y b$-EGFP positive cells. As expected, a much lower methylation status was found in these four CpG islands in MO-injected cmyb-EGFP positive cells (Figure 4C, Additional file 1: Figure S6). Taken together, cebpa expression is likely regulated by Dnmt1 activity.

\section{Cebpa upregulation was involved in the defective HSPC phenotypes in dnmt1-deficient embryos}

A series of rescue assays were carried out in order to verify whether elevated expression of cebpa is responsible for dnmt1 mutant phenotype. In our previous work, two repressive forms of C/ebpa have been constructed [49], SUMO2-C/ebpa, mimicking the constitutively sumoylated form of C/ebpa and POZ-C/ebpa, mimicking the SUMO-mediated repressive form. The mRNAs of these two constructs were individually injected into the wild-type embryos with dnmt1 ATG
$\mathrm{MO}$, respectively. Both were shown to be effective in rescuing $c m y b$ expression (Figure $5 \mathrm{~A}-\mathrm{H}$ ) in the morphants. The similar rescuing results were also observed in ldd794 mutant embryos overexpressing SUMO2-C/ ebpa repressive protein (Figure 5I-N).

It is worth noting that two dnmt1 mutant lines with liver and pancreas development defects were reported [50]. As expected, the similar phenotypes were also detected in our ldd794 mutants by assessing the expression of hepatocytes marker lfabp and pancreas marker trypsin (Additional file 1: Figure S7). Intriguingly, the rescue effects of SUMO2-C/ebpa on liver and pancreas development defects were not observed (Additional file 1: Figure S7), suggesting the role of Dnmt1 in liver and pancreas development was unlikely cebpa dependent.

Taken together, these data strongly suggest that elevated cebpa function is involved in HSPC defects of dnmt1 mutants.

\section{Cebpa function was pivotal to Dnmt1 regulated maintenance of definitive HSPCs}

We have generated a cebpa null mutant zebrafish line by TALEN approach (submitted elsewhere). The phenotype was similar to that observed in mice. While no mature 


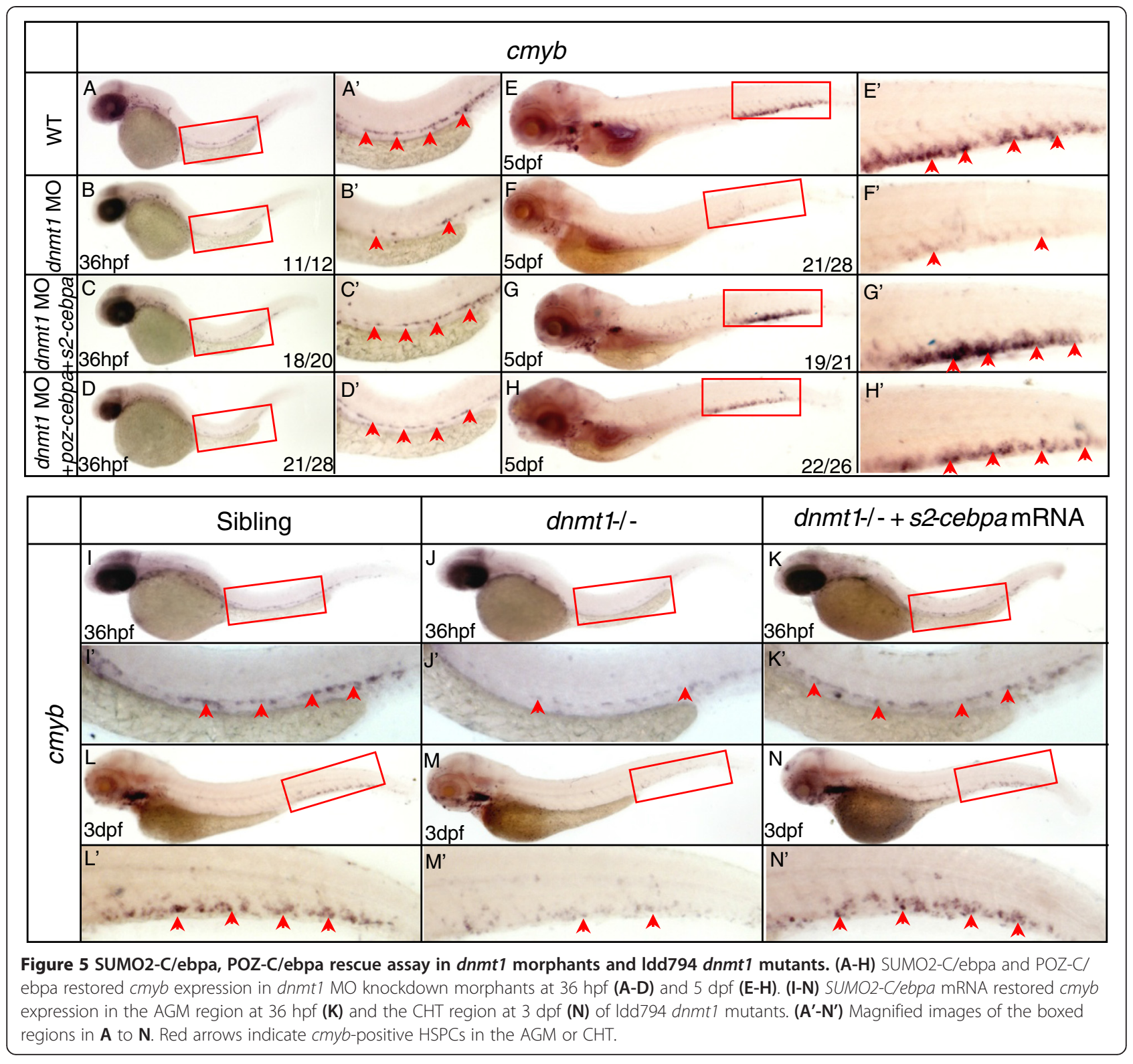

myelocytes were detected by Sudan Black staining, the expression of cmyb remained unchanged, if not a marginal increase. This cebpa mutant line was then crossed with ldd794 heterozygotes. The adult fishes carrying both cebpa and dnmt1 mutant alleles were incrossed. We found only in the presence of C/ebpa that cmyb expression was severely decreased (Figure 6B, B'). By contrast, in the cebpa and dnmt1 double null mutants, the expression level of cmyb remained normal (Figure 6D, D'). In parallel, similar results were observed in cebpa null embryos knocked down with dnmt1 MO (Additional file 1: Figure S8). These results indicate that the defective HSPCs triggered by dnmt1 mutation require intact $\mathrm{C} / \mathrm{ebpa}$ function. Therefore, cebpa is a key target gene of $d n m t 1$ and its regulated function is involved in the definitive HSPCs maintenance.

\section{Discussion}

In this study, a zebrafish mutant line with definitive hematopoiesis defects was identified. The specific phenotype was caused by a premature termination codon in the dnmt1 gene, which resulted in a truncated Dnmt1 protein lacking its catalytic domain. DNMT1 protein is an important DNA methyltransferase to silence and regulate genes by methylation of DNA regions without changing the genomic DNA sequence [23]. Lack of Dnmt1 function resulted HSPC proliferation block and shortage of differentiated blood lineages. We demonstrated that normal Dnmt1 function was critical in regulating cebpa gene expression, and intact C/ebpa function was required for HSPC proliferation block triggered by the absence of Dnmt1 function. Our 


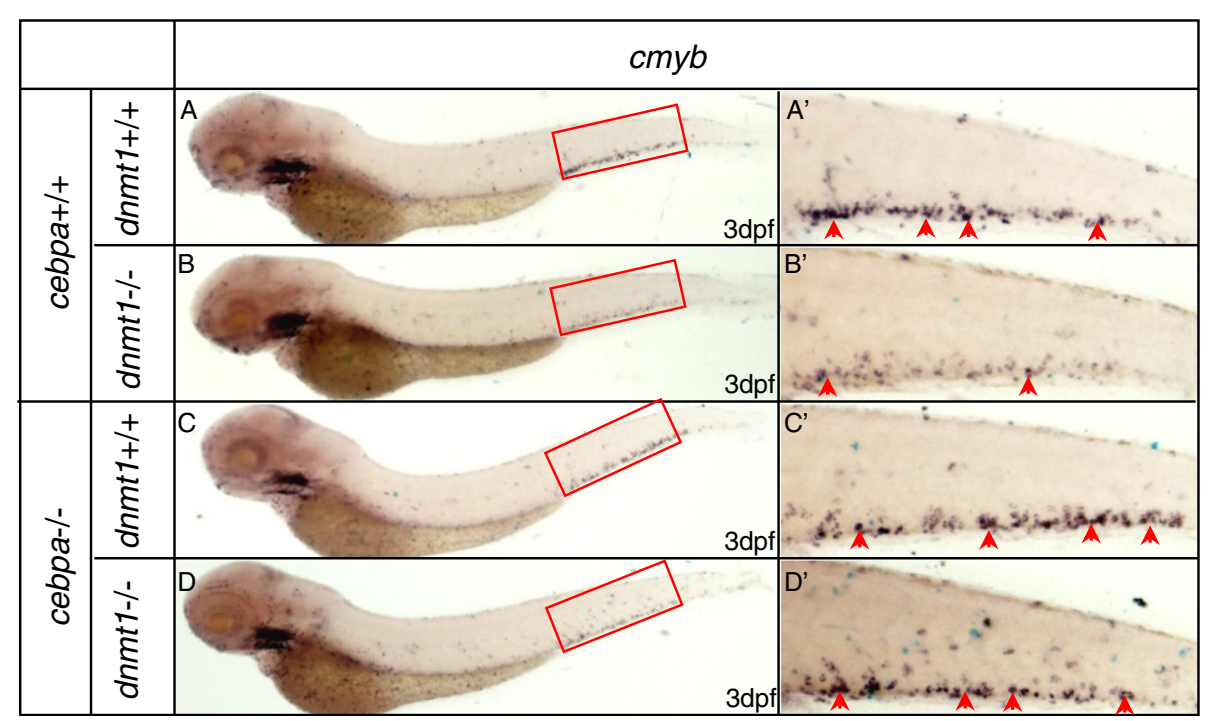

Figure 6 WISH assays of $c m y b$ in cebpa null mutants and siblings with or without dnmt 1 mutation. (A) WISH assay of cmyb in embryos without cebpa and dnmt1 homozygote mutation. (B) WISH assay of cmyb in embryos only with dnmt1 homozygote mutation. Note that $\mathrm{cmyb}$ was markedly decreased. (C) WISH assay of cmyb in embryos only with cebpa homozygote mutation. (D) WISH assay of cmyb in embryos both with cebpa and dnmt1 homozygote mutation. Note that cmyb was comparable with embryos without dnmt1 homozygote mutation. $\left(\mathbf{A}^{\prime}-\mathbf{D}^{\prime}\right)$ Magnified images of the boxed regions in $\mathbf{A}$ to $\mathbf{D}$. Red arrows indicate cmyb-positive HSPCs in the CHT.

studies provided new evidence for that cebpa is a downstream target of Dnmt1 in regulating HSPC proliferation during normal hematopoiesis.

Many lines of evidences demonstrate that DNA methylation influences gene expression during embryogenesis [51-53]. In mice, a Dnmt1 mutation led to a recessive lethal phenotype with stunted and delayed development [24]. Similarly, two other zebrafish mutants related to dnmt1 were shown to have defects in pancreas development at a late embryonic stage and resulted in embryonic lethality [50]. In line with these mutant phenotypes, our ldd794 homozygous mutants usually die at $8 \mathrm{dpf}$ with abnormal hematopoiesis and other organ formation such as the liver, further suggesting that reduced DNA methylation causes developmental abnormality and embryonic lethality. It is worth noting that ldd794 heterozygotes do not display any observable phenotype, implying that the mutated dnmt 1 allele does not play a dominant negative role.

Our developmental and molecular analyses showed that the lack of Dnmt1 enzymatic activity in ldd794 mutants led to severe reduction in HSPC numbers as well as impaired production of all three major lineages but accompanied by normal vascular development during early development. These observations are in an agreement with the ones from mouse studies [28]. Bone marrow transplant assays revealed that Dnmt1 affected HSCs in a cell-autonomous manner [28]. Our findings, together with those in mice, demonstrate that Dnmt1 has a conserved role in definitive hematopoiesis.
The phenotype with lower number of HSPCs in CHT might be due to either reduced proliferation or increased apoptosis of HSPCs. The pH3 and TUNEL assays suggested that the definitive HSPC defects are not due to increased apoptosis but likely caused by decreased proliferation of HSPCs. These results are also consistent with the fact that the frequencies of apoptotic cells in total BM, CMPs, GMPs, or MEPs remained unchanged in mouse mutants [28].

Given the fact that C/ebpa is a critical transcription factor for granulopoiesis [43], we have expected that its activation might induce accelerated myeloid differentiation. However, downregulated $m p x$ expression in dnmt1 mutant does not support an increased myeloid differentiation process. Meanwhile, an increased number of reports have demonstrated that C/ebpa is an important modulator of HSPC function [44-47]. Supporting this idea, we have found that hypomethylation of the cebpa regulatory region as a result of Dnmt1 deficiency is directly associated with HSPC impairment. Although the possibility of other negative regulators being activated cannot be excluded completely, HSPC proliferation defect caused by dnmt1 mutation is indeed C/ebpa dependent; as in the cebpal dnmt1 double null mutants, the $c m y b$ expression appeared to be normal. It was reported that C/EBPa negatively regulated n-myc, and the loss of C/EBPa resulted in de-repression of n-myc in mice HSCs [47]. Indeed, our Q-PCR analysis revealed that in $c m y b$-EGFP positive cells sorted from dnmt1 MO knockdown embryos, $n$-myc had a much lower expression level (data not shown), which 
might account for the pronounced decreased proliferation of HSPCs. Finally, one recent report revealed that a non-coding RNA arising from the CEBPA gene locus could influence the methylation level of $C E B P A$ promoter by inhibiting DNMT1 protein binding to the regulatory region of CEBPA gene [54], which also supported our findings that Dnmt1 acts directly on cebpa promoter.

Taken together, our findings and others point out that the regulation of C/ebpa function during hematopoiesis takes place at multi-levels, including epigenetic modification, transcriptional regulation, and post-translational modification, which allow C/ebpa to exert its distinguished role in a fine-tuned manner.

\section{Conclusions}

Our studies for the first time clarify the possible molecular mechanism of Dnmt1 involved in HSPC maintenance during definitive hematopoiesis in zebrafish. The fact that C/ebpa functions as a critical downstream effector of Dnmt1 provides new insights of Dnmt1-regulated hematopoiesis.

\section{Methods}

\section{Zebrafish maintenance and ENU mutagenesis}

Zebrafish were maintained and staged under standard conditions as described previously [55]. Zebrafish embryos were cultured in "egg water" consisting of $0.03 \%$ sea salt and $0.002 \%$ methylene blue. A $0.0045 \%$ 1-phenyl-2-thiourea (Sigma-Aldrich, St. Louis, MO, USA) was used to prevent melanization and facilitate in situ hybridization analysis of gene expression [55]. ENU mutagenesis on Tubingen (Tu) strain was carried out as described [56]. The WIK line was used as the mapping strain. The zebrafish maintenance and study protocols were approved by the Institutional Review Board of the Institute of Health Sciences, Shanghai Institutes of Biological Sciences, Chinese Academy of Sciences (Shanghai, China).

\section{Whole-mount in situ hybridization (WISH)}

Digoxigenin (DIG)-labeled RNA probes were synthesized with T3 or T7 polymerase (Ambion, Life Technologies, Carlsbad, CA, USA), using linearized cDNA plamid constructs. Whole-mount mRNA in situ hybridization was performed as described previously [57]. The DIG-labeled probes were detected using alkaline phosphatase-coupled anti-digoxigenin Fab fragment antibody (Roche, Basel, Switzerland) with BCIP/NBT staining (Vector Laboratories, Burlingame, CA, USA).

\section{Mapping and positional cloning of Idd794}

ldd794 ( $\mathrm{Tu}$ background) carrying the mutant allele were outcrossed to the polymorphic wild-type strain WIK for positional cloning. The genome for linked SSLP (simple sequence length polymorphism) markers were scanned by bulk segregation analysis using standard methods [58]. For fine mapping, ldd794 mutant embryos were genotyped with SSLP markers to narrow down the genetic interval. The cDNAs of candidate genes were sequenced from pooled mutant RNA, and candidate mutation was confirmed by sequencing the genomic DNA of individual mutant embryo. All primers used for positional cloning and $d n m t 1$ sequencing are provided in Additional file 2.

\section{Morpholinos and mRNA microinjection}

Morpholinos (MOs) and mRNA were injected into embryos at one-cell stage. Morpholino oligonucleotides were designed by and ordered from Gene Tools. The morpholino sequences are as follows: for dnmt1 MO, 5'-ACAAT GAGGTCTTGGTAGGCATTTC-3' (4 ng/embryo) [27]; and for dnmt1 splicing MO, 5'-CCACCCTTCAAAA CAATAACAGTGT-3' (8 ng/embryo). Capped mRNA samples were transcribed from linearized plasmids (mMessage Machine; Ambion), purified, and diluted to $100 \mathrm{ng} / \mathrm{ul}$ (dnmt1 and dnmt1 mutant mRNA) or $50 \mathrm{ng} / \mathrm{ul}$ (SUMO2-C/ebpa and POZ-C/ebpa mRNA) for injection of embryos at one-cell stage.

\section{Anti-phosphorylated histone $\mathrm{H} 3$ (pH3) immunostaining and TUNEL assay}

Three days post-fertilization (dpf), Tg (cmyb:eGFP) embryos were fixed in $4 \%$ paraformaldehyde (PFA). After dehydration and rehydration, the embryos were treated with Proteinase $\mathrm{K}(10 \mathrm{mg} / \mathrm{ml})$ for $30 \mathrm{~min}$ at RT and refixed in 4\% PFA for $20 \mathrm{~min}$. After blocking with blocking buffer $(2 \mathrm{mg} / \mathrm{ml} \mathrm{BSA}+10 \% \mathrm{FBS}+0.3 \%$ Triton-X100 + 1\% DMSO in PBST), the embryos were stained with mouse anti-GFP (Invitrogen, Carlsbad, CA, USA) and rabbit anti-phosphohistone $\mathrm{H} 3$ antibody (Santa Cruz) primary antibody at $4^{\circ} \mathrm{C}$ overnight. Alexa Fluor 488conjugated anti-mouse (Invitrogen) and Alexa Fluor 594-conjugated anti-rabbit (Invitrogen) were used as secondary antibodies. Images were taken using Olympus FV 1000 confocal microscopy equipped with the FV10-ASW version 3 software.

Terminal transferase UTP nick end labeling (TUNEL) was performed using the In Situ Cell Death Detection Kit, TMR red (Roche), according to the manufacturer's recommendations.

\section{Genomic DNA and RNA isolation}

$\mathrm{Tg}$ (cmyb:EGFP) embryos at one-cell stage were injected with dnmt1 ATG morpholino. Cells positive for $c m y b$-EGFP were sorted and collected from homogenized embryos at $3 \mathrm{dpf}$. Genomic DNA (gDNA) and total mRNA were extracted using the TRIzol reagent (Life Technologies) according to the manufacturer's instructions. 


\section{Quantitative real-time PCR}

Reverse transcription was carried out using the super script first-strand synthesis system (Life Technologies) according to the manufacturer's instructions. Real-time quantitative PCR (Applied Biosystems, Foster City, CA, USA) was used for relative quantification of cebpa gene expression. The expression level of cebpa was normalized to the expression of housekeeping gene GAPDH. The primers used for real-time quantitative PCR were listed in Additional file 2.

\section{Bisulfite sequencing PCR (BSP) assay}

The DNA methylation assay was performed using the EZ DNA Methylation Kit (Zymo Research, Irvine, CA, USA) according to the manufacturer's recommendations. The treatment of DNA with bisulfite results in the selective conversion of unmethylated cytosine to uracil, whereas methylated cytosine remains unchanged. Methprimer (http://www.urogene.org/methprimer/) was used to predict the CpG islands. The following primers were used for bisulfite-specific polymerase chain reaction of the regulation regions of the cebpa gene: BSP1 Fw: 5'-GTTTTATA GAAGTTTGTTAGAGGGG-3' and Rv: $5^{\prime}$-AACAAACC CAACCCTTCTTTATTAT-3'; BSP2 Fw: 5'-TTTTTTT TAGATGGTTTGTTTTAGG-3' and Rv: 5'-ATAAATT CACCCAAAAATTCAAAAC-3'; BSP3 Fw: 5'-TTTGA TAATTAGTATGAATTGTTTTGTTTT-3' and RV: 5'AACTTTAACCATATTATCCAAAATCACAT-3'; BSP4 Fw: 5' -ATATTTTTTGTGTAGATTTAAAATGGTGTT$3^{\prime}$ and Rv: 5'-TACTCCATATAACACATTTAATCCAA CTAA-3'. PCR products were subcloned into pMD18-T Vector (Takara, Kyoto, Japan), and transformed bacteria were cultured overnight. Clones (eight to ten) of each BSP were sequenced for confirmation.

\section{Additional files}

Additional file 1: Supplementary figures and figure legends.

Additional file 2: Table S1. The sequence information of SSLP markers, dnmt1 sequencing primers, and Q-PCR primers.

\begin{abstract}
Abbreviations
Dnmt1: DNA methyltransferase 1; HSPCs: Hematopoietic stem and progenitor cells; C/ebpa: CCAAT/enhancer-binding protein alpha (zebrafish gene: cebpa); C/EBPa: Mouse gene; C/EBPa: Mouse protein; C/EBPA: Human gene; C/EBPA: Human protein; HSCs: Hematopoietic stem cells; VDA: Ventral wall of the dorsal aorta; AGM: Aorta/gonad/mesonephros; CHT: Caudal hematopoietic tissue; AML: Acute myeloid leukemia; LSC: Leukemia stem cell; ENU: N-ethyl-N-nitrosourea; WISH: Whole-mount mRNA in situ hybridization; hpf: Hours post-fertilization; dpf: Days postfertilization; pH3: Phosphorylated histone H3; TUNEL: Terminal transferase UTP nick end labeling; BSP: Bisulfite sequencing PCR.
\end{abstract}

\section{Competing interests}

The authors declare that they have no competing interests.

\section{Authors' contributions}

$X L$ performed the experiments and analyzed the data. XJ, YX, HY, KM, YC, and $Y J$ assisted with the experiments. MD, WP, SC, ZC, HdT, LZ, YZ, JZ, and $J Z$ designed the research plan. JZ and JZ wrote the paper. All authors read and approved the final manuscript.

\section{Acknowledgements}

We thank Dr. David A. Jones for providing the dnmt1 plasmid. We also thank Juan Chen and Wu Zhang for cell sorting.

\section{Funding}

This study was supported by research funding from the National Basic Research Program of China (2012CB910300), National Natural Science Foundation of China (81300372), Program for Professor of Special Appointment (Eastern Scholar) at Shanghai Institutions of Higher Learning, and Doctoral Innovation Fund Project from Shanghai Jiao Tong University School of Medicine (BXJ201315).

\section{Author details}

${ }^{1}$ CNRS-LIA124, Sino-French Research Center for Life Sciences and Genomics, State Key Laboratory of Medical Genomics, Rui Jin Hospital, Shanghai Jiao Tong University School of Medicine, Shanghai 200025, China. ${ }^{2}$ Key Laboratory of Stem Cell Biology, Institute of Health Sciences, Shanghai Institutes for Biological Sciences, Chinese Academy of Sciences \& Shanghai Jiao Tong University School of Medicine, Shanghai 200031, China.

${ }^{3}$ Laboratory of Development and Diseases, State Key Laboratory for Medical Genomics, Shanghai Institute of Hematology, Rui Jin Hospital, Shanghai Jiao Tong University School of Medicine, Shanghai 200025, China. ${ }^{4}$ Institute of Health Sciences, Shanghai Institutes for Biological Sciences, Chinese Academy of Sciences \& Shanghai Jiao Tong University School of Medicine, Shanghai 200031, China. ${ }^{5}$ Equipe Labellisée No. 11 Lique Nationale Contre le Cancer, Hôpital St. Louis, Université de Paris 7/INSERM/CNRS UMR 944/7212, 75475 Paris, France. ${ }^{6}$ Stem Cell Program, Hematology/Oncology Program at Children's Hospital Boston, Harvard Medical School, Boston, MA 02114, USA.

Received: 4 November 2014 Accepted: 24 January 2015

Published online: 22 February 2015

\section{References}

1. Galloway JL, Zon LI. Ontogeny of hematopoiesis: examining the emergence of hematopoietic cells in the vertebrate embryo. Curr Top Dev Biol. 2003;53:139-58.

2. Zon LI. Developmental biology of hematopoiesis. Blood. 1995;86:2876-91.

3. Haar JL, Ackerman GA. A phase and electron microscopic study of vasculogenesis and erythropoiesis in the yolk sac of the mouse. Anat Rec. 1971:170:199-223.

4. Takahashi K, Yamamura F, Naito M. Differentiation, maturation, and proliferation of macrophages in the mouse yolk sac: a light-microscopic, enzyme-cytochemical, immunohistochemical, and ultrastructural study. J Leukoc Biol. 1989:45:87-96.

5. de Jong JL, Zon LI. Use of the zebrafish system to study primitive and definitive hematopoiesis. Annu Rev Genet. 2005;39:481-501.

6. Carradice D, Lieschke GJ. Zebrafish in hematology: sushi or science? Blood. 2008;111:3331-42.

7. Song HD, Sun XJ, Deng M, Zhang GW, Zhou Y, Wu XY, et al. Hematopoietic gene expression profile in zebrafish kidney marrow. Proc Natl Acad Sci U S A. 2004;101:16240-5.

8. Ransom DG, Haffter P, Odenthal J, Brownlie A, Vogelsang E, Kelsh RN, et al. Characterization of zebrafish mutants with defects in embryonic hematopoiesis. Development. 1996;123:311-9.

9. Weinstein BM, Schier AF, Abdelilah S, Malicki J, Solnica-Krezel L, Stemple DL, et al. Hematopoietic mutations in the zebrafish. Development. 1996;123:303-9.

10. Bertrand JY, Chi NC, Santoso B, Teng S, Stainier DY, Traver D. Haematopoietic stem cells derive directly from aortic endothelium during development. Nature. 2010:464:108-11.

11. Kissa K, Herbomel P. Blood stem cells emerge from aortic endothelium by a novel type of cell transition. Nature. 2010:464:112-5.

12. Bertrand JY, Kim AD, Teng S, Traver D. CD41+ cmyb + precursors colonize the zebrafish pronephros by a novel migration route to initiate adult hematopoiesis. Development. 2008;135:1853-62. 
13. Rice KL, Hormaeche I, Licht JD. Epigenetic regulation of normal and malignant hematopoiesis. Oncogene. 2007;26:6697-714.

14. Li E, Beard C, Jaenisch R. Role for DNA methylation in genomic imprinting. Nature. 1993;366:362-5.

15. Panning $B$, Jaenisch $R$. DNA hypomethylation can activate Xist expression and silence X-linked genes. Genes Dev. 1996;10:1991-2002.

16. Chen RZ, Pettersson U, Beard C, Jackson-Grusby L, Jaenisch R. DNA hypomethylation leads to elevated mutation rates. Nature. 1998;395:89-93.

17. Li KK, Luo LF, Shen Y, Xu J, Chen Z, Chen SJ. DNA methyltransferases in hematologic malignancies. Semin Hematol. 2013;50:48-60.

18. Yen RW, Vertino PM, Nelkin BD, Yu JJ, el-Deiry W, Cumaraswamy A, et al. Isolation and characterization of the cDNA encoding human DNA methyltransferase. Nucleic Acids Res. 1992;20:2287-91.

19. Okano M, Xie S, Li E. Cloning and characterization of a family of novel mammalian DNA (cytosine-5) methyltransferases. Nat Genet. 1998;19:219-20.

20. Xie S, Wang Z, Okano M, Nogami M, Li Y, He WW, et al. Cloning, expression and chromosome locations of the human DNMT3 gene family. Gene. 1999;236:87-95.

21. Jurkowska RZ, Jurkowski TP, Jeltsch A. Structure and function of mammalian DNA methyltransferases. Chembiochem. 2011;12:206-22.

22. Lei H, Oh SP, Okano M, Juttermann R, Goss KA, Jaenisch R, et al. De novo DNA cytosine methyltransferase activities in mouse embryonic stem cells. Development. 1996;122:3195-205.

23. Singal R, Ginder GD. DNA methylation. Blood. 1999;93:4059-70.

24. Li E, Bestor TH, Jaenisch R. Targeted mutation of the DNA methyltransferase gene results in embryonic lethality. Cell. 1992;69:915-26.

25. Stancheva I, Hensey C, Meehan RR. Loss of the maintenance methyltransferase, xDnmt1, induces apoptosis in Xenopus embryos. EMBO J. 2001;20:1963-73.

26. Martin CC, Laforest L, Akimenko MA, Ekker M. A role for DNA methylation in gastrulation and somite patterning. Dev Biol. 1999;206:189-205.

27. Rai K, Nadauld LD, Chidester S, Manos EJ, James SR, Karpf AR, et al. Zebra fish Dnmt1 and Suv39h1 regulate organ-specific terminal differentiation during development. Mol Cell Biol. 2006;26:7077-85

28. Trowbridge JJ, Snow JW, Kim J, Orkin SH. DNA methyltransferase 1 is essential for and uniquely regulates hematopoietic stem and progenitor cells. Cell Stem Cell. 2009;5:442-9.

29. Mizuno S, Chijiwa T, Okamura T, Akashi K, Fukumaki Y, Niho Y, et al. Expression of DNA methyltransferases DNMT1, 3A, and 3B in normal hematopoiesis and in acute and chronic myelogenous leukemia. Blood. 2001;97:1172-9.

30. Trowbridge JJ, Sinha AU, Zhu N, Li M, Armstrong SA, Orkin SH. Haploinsufficiency of Dnmt1 impairs leukemia stem cell function through derepression of bivalent chromatin domains. Genes Dev. 2012;26:344-9.

31. Thompson MA, Ransom DG, Pratt SJ, MacLennan H, Kieran MW, Detrich 3rd $H W$, et al. The cloche and spadetail genes differentially affect hematopoiesis and vasculogenesis. Dev Biol. 1998;197:248-69.

32. North TE, Goessling W, Walkley CR, Lengerke C, Kopani KR, Lord AM, et al. Prostaglandin E2 regulates vertebrate haematopoietic stem cell homeostasis. Nature. 2007;447:1007-11.

33. Detrich 3rd HW, Kieran MW, Chan FY, Barone LM, Yee K, Rundstadler JA, et al. Intraembryonic hematopoietic cell migration during vertebrate development. Proc Natl Acad Sci U S A. 1995;92:10713-7.

34. Brownlie A, Hersey C, Oates AC, Paw BH, Falick AM, Witkowska HE, et al. Characterization of embryonic globin genes of the zebrafish. Dev Biol. 2003;255:48-61.

35. Lieschke GJ, Oates AC, Crowhurst MO, Ward AC, Layton JE. Morphologic and functional characterization of granulocytes and macrophages in embryonic and adult zebrafish. Blood. 2001;98:3087-96.

36. Berman JN, Kanki JP, Look AT. Zebrafish as a model for myelopoiesis during embryogenesis. Exp Hematol. 2005;33:997-1006.

37. Meijer AH, van der Sar AM, Cunha C, Lamers GE, Laplante MA, Kikuta H, et al. Identification and real-time imaging of a myc-expressing neutrophil population involved in inflammation and mycobacterial granuloma formation in zebrafish. Dev Comp Immunol. 2008;32:36-49.

38. Willett CE, Cherry JJ, Steiner LA. Characterization and expression of the recombination activating genes (rag1 and rag2) of zebrafish. Immunogenetics. 1997;45:394-404.

39. Boisset JC, van Cappellen W, Andrieu-Soler C, Galjart N, Dzierzak E, Robin C. In vivo imaging of haematopoietic cells emerging from the mouse aortic endothelium. Nature. 2010;464:116-20.
40. Lam EY, Hall CJ, Crosier PS, Crosier KE, Flores MV. Live imaging of Runx1 expression in the dorsal aorta tracks the emergence of blood progenitors from endothelial cells. Blood. 2010;116:909-14.

41. Landschulz WH, Johnson PF, Adashi EY, Graves BJ, McKnight SL. Isolation of a recombinant copy of the gene encoding C/EBP. Genes Dev. 1988;2:786-800.

42. Landschulz WH, Johnson PF, McKnight SL. The leucine zipper: a hypothetical structure common to a new class of DNA binding proteins. Science. 1988;240:1759-64

43. Radomska HS, Huettner CS, Zhang P, Cheng T, Scadden DT, Tenen DG CCAAT/enhancer binding protein alpha is a regulatory switch sufficient for induction of granulocytic development from bipotential myeloid progenitors. Mol Cell Biol. 1998;18:4301-14.

44. Zhang P, Iwasaki-Arai J, Iwasaki H, Fenyus ML, Dayaram T, Owens BM, et al, Enhancement of hematopoietic stem cell repopulating capacity and selfrenewal in the absence of the transcription factor C/EBP alpha. Immunity. 2004;21:853-63.

45. Heath V, Suh HC, Holman M, Renn K, Gooya JM, Parkin S, et al. C/EBPalpha deficiency results in hyperproliferation of hematopoietic progenitor cells and disrupts macrophage development in vitro and in vivo. Blood. 2004;104:1639-47.

46. Fukuchi $Y$, Ito M, Shibata F, Kitamura T, Nakajima H. Activation of CCAAT/ enhancer-binding protein alpha or PU.1 in hematopoietic stem cells leads to their reduced self-renewal and proliferation. Stem Cells. 2008;26:3172-81.

47. Ye M, Zhang $H$, Amabile G, Yang H, Staber PB, Zhang P, et al. C/EBPa controls acquisition and maintenance of adult haematopoietic stem cell quiescence. Nat Cell Biol. 2013;15:385-94.

48. Johnson PF. Molecular stop signs: regulation of cell-cycle arrest by C/EBP transcription factors. J Cell Sci. 2005;118:2545-55.

49. Yuan H, Zhou J, Deng M, Zhang Y, Chen Y, Jin Y, et al. Sumoylation of CCAAT/enhancer-binding protein alpha promotes the biased primitive hematopoiesis of zebrafish. Blood. 2011;117:7014-20.

50. Anderson RM, Bosch JA, Goll MG, Hesselson D, Dong PD, Shin D, et al. Loss of Dnmt1 catalytic activity reveals multiple roles for DNA methylation during pancreas development and regeneration. Dev Biol. 2009;334:213-23.

51. Shemer R, Kafri T, O'Connell A, Eisenberg S, Breslow JL, Razin A. Methylation changes in the apolipoprotein Al gene during embryonic development of the mouse. Proc Natl Acad Sci U S A. 1991;88:11300-4.

52. Sanosaka T, Tsujimura K. Nakashima K [Epigenetic regulation involved in fate specification of neural cells]. Tanpakushitsu Kakusan Koso. 2008;53:331-7.

53. Nishino K, Hattori N, Tanaka S, Shiota K. DNA methylation-mediated control of Sry gene expression in mouse gonadal development. J Biol Chem. 2004;279:22306-13.

54. Di Ruscio A, Ebralidze AK, Benoukraf T, Amabile G, Goff LA, Terragni J, et al DNMT1-interacting RNAs block gene-specific DNA methylation. Nature. 2013;503:371-6.

55. Kimmel CB, Ballard WW, Kimmel SR, Ullmann B, Schilling TF. Stages of embryonic development of the zebrafish. Dev Dyn. 1995;203:253-310.

56. Mullins MC, Nusslein-Volhard C. Mutational approaches to studying embryonic pattern formation in the zebrafish. Curr Opin Genet Dev. 1993;3:648-54.

57. Thisse C, Thisse B. High-resolution in situ hybridization to whole-mount zebrafish embryos. Nat Protoc. 2008;3:59-69.

58. Bahary N, Davidson A, Ransom D, Shepard J, Stern H, Trede N, et al. The Zon laboratory guide to positional cloning in zebrafish. Methods Cell Biol. 2004;77:305-29.

\section{Submit your next manuscript to BioMed Central and take full advantage of:}

- Convenient online submission

- Thorough peer review

- No space constraints or color figure charges

- Immediate publication on acceptance

- Inclusion in PubMed, CAS, Scopus and Google Scholar

- Research which is freely available for redistribution 